Available online at https://www.irjst.com/
International Research Journal of Science and Technology
ISSN:2707-3955
DOI: https://doi.org/10.46378/irjst.2020.010206

\title{
A Bio-Inspired Reward-Based Message Forwarding For Vehicular Social Network
}

\section{Aklilu Mandefro Messele \\ Department of Computer Science, Mega Computer and Research Center, Gondar, Ethiopia}

\begin{tabular}{|c|c|}
\hline Paper Status & \\
\hline Received : & January 2020 \\
\hline Accepted & March 2020 \\
\hline Published & March 2020 \\
\hline
\end{tabular}

Key Words

Bio-Inspired

Cooperative

Paying Per Packet

Reward

Selfishness

\section{Abstract}

The Ants capability of working together and their way of message transmission makes us inspired especially their cooperation for their common goal and dropping down pheromones which used as a trail for themselves and for others. We would use Ants broadcasting method for drivers' cooperation and message forwarding. Therefore if there were any driver who couldn't broadcast any emergency message related to car accident like Ants done to locate the food source, could be considered as a selfish node and couldn't be rewarded as cooperatives do. The previous vehicle to vehicle communication or message forwarding schemes that was tried to motivate cooperative drivers used incentive methods in which it is not motivating all participant nodes and some of them used punishment methods. These types of methods are not effective to stimulate selfish drivers. We conducted the research using embedded traffic simulation in which it includes mobility and network simulation capabilities merged together and served as multi-functional software. From the tools falling into the embedded category is National Chiao Tung University network simulator (NCTUns) which provides a full range of network stack simulation tools. Watchdog was our selfishness detection method, in which the RSU controls whether nodes are cooperative or not because the (Road Side Unit) RSU knows where the nodes were located and in which path they are drive and what messages they broadcasted. The rewarding model we used is paying per packet. The payment principle is for each successfully transmitted unit-sized packet, each of $\mathrm{N}$ intermediate nodes should receive $\lambda$ credits, whereas the service provider (SP) would pay $\lambda * \mathrm{~N}$ in total. The proposed solution was evaluated by the three performance metrics which are dropped packets, selfishness detection method and delivery ratio. Based on our experiments result the number of accident increased as the number of total nodes increased in the same simulation area. As the number of nodes participated on the area increases the probability of having an accident increases and the probability of stimulating selfish nodes increased using the same incentive method and amount because there is a probability of accident commit increases and the rewarding and acknowledgement messages also increases in which it can stimulate the selfish nodes.

Copyright (C) 2020: Aklilu Mandefro Messele. This is an open access distribution, and reproduction in any medium, provided Access article distributed under the Creative Commons Attribution License the original work is properly cited License, which permits unrestricted use.

Citation: Aklilu Mandefro Messele. “A Bio-Inspired Reward-Based Message Forwarding For Vehicular Social Network", International Research Journal of Science and Technology, 1 (2) 106-119, 2020.

\section{Introduction}

Vehicular social networks, is the combination of Social characteristics and human behavior of individual vehicles "socializing" and sharing messages based on their common interests. "The concept of a social car arises from the assumption that each driver can share data with other neighbors based on common interests" [1]. Many scholars and researchers were and are interested in the behaviors of animals' especially social insects for many different purposes. Nowadays researchers on computer science and information 
technology are also interested and published papers which are related to nature and social insects mostly honey Bees and Ants [12].

The multimodal communication which includes tactile, acoustic, visual, sometimes magnetic and especially chemical signals are ways of optional communication systems among social insects. Due to these multimodal communication signals is considered as the reason for their social organization [2]. Pheromone is a chemical signal transmitted between members of the same species [3].

Ants are walking randomly for searching food and when they back to their nest they marked with a pheromone trail and they return to their nest following the trail. During returning, they produced an extensive pheromone trail to pointing towards the food source. Therefore ants are communicating based on environmental changes or pheromone trail, i.e. they use stigmergic or chemical communication techniques for communication and collaboration [4]. Even if, they use trail because of the worker Ants do not seem to be guided by their own memory or landmarks [5], this pheromonal message is visible for all Ants and they can take an immediate measurement by using the broadcasted message. There may be warning pheromone and food source locating pheromone. Whatever the pheromone is we are interested on their message broadcasting for their common interest. Even they can establish pheromonal trail with the presence of obstacles [6]. Ants have a capability of selecting a shortest path while back from the source node to the nest. Because of Ants considered the pheromone with the greatest density as a shortest path and the better food source [7], then Ants follow this path during collecting their food from the selected source [8], this indicates Ants use two pheromonal chemical one from the nest to food source and the other is back from the food source to the nest [6].

The most widespread communication form among social insects is chemical messenger molecule which is known as 'pheromone'. This pheromone is a chemical composition of pheromonal secretions which is corresponds with simple mixtures of small and volatile molecules then due to pheromones evaporation or fadeout, its concentration decreases over time and distance. So every community of Ants can be well informed because of they are broadcasting every messages on their path.

\footnotetext{
* Corresponding author: Aklilu Mandefro Messele1

Department of Computer Science, Bahirdar University, Ethiopia

Email: bepositive2712@yahoo.com,aklilu.mandefro@yahoo.com
}

This is what many of human beings lack. Therefore there have to be the mechanism that can decrease the selfishness of human beings and increase our cooperative. That is rewarding drivers with good behaviors so as to attract the selfish drivers [2].

The Ants capability of working together and their way of message transmission makes us inspired especially their cooperation for their common goal and dropping down pheromones which used as a trail for themselves and for others. Therefore by keeping this Ants behavior of cooperation and pheromonal message forwarding, we would use for drivers cooperation and message forwarding. Therefore if there were any driver who couldn't broadcast any emergency message related to car accident like Ants done to locate the food source, could be considered as a selfish node and couldn't be rewarded as cooperatives do.

Selfishness may be classified as mass selfishness and individual selfishness. Mass selfishness can be defined that every node has the selfishness nature so that it may probabilistically drop a certain percentage of messages instead of forwarding it. On the other hand, in an individual selfishness case, only a small number of selfish nodes may not be willing to forward packets for others. So the selfish drivers in the vehicles may not want to forward the packets if it will not benefit them in some way [9]. The existence of some selfish nodes, especially in a network that needs cooperation like DTN, could cause catastrophic damage to any well designed opportunistic routing scheme and endanger the whole network [10].

When we see the Rewarding Model, There are several available rewarding models that can be adopted in previous works For example, a popular charging method in Y. Zhang et al., [9] A secure incentive protocol for mobile ad hoc networks is paying per packet, which means that, for each successfully transmitted unit-sized packet, each of $\mathrm{N}$ intermediate nodes should receive $\lambda$ credits, whereas the source needs to pay $\lambda * \mathrm{~N}$ in total the internet service provider is going to pay the credit or the reward for the nodes cooperation. Even if some of the researchers try to argue that this method is not suitable for opportunistic data forwarding in that it is difficult for the source to predict how many copies or hops are needed to successfully deliver a message to the destination but the number of intermediate nodes in the forwarding process is not a headache for our purpose because the reward is not given by the source node instead the source node is also from the nodes that have to get a reward from the service provider. Therefore, in our case paying per packet would use as a rewarding model. We care on the rewarding model because the design of the reward calculation is the core of the 
incentive scheme as it stated by the same researcher to avoid repetitiveness or forwarding the same message more than Vehicles that had to ignore a packet if they have received it before. Fairness is guaranteed because only nodes that participate in the message forwarding would reward.

In this bio-inspired reward-based message forwarding research work, forwarding a message from the source node to the destination is not a must and not necessarily needed because mostly car drivers which are located around the car accident have to get messages related to the accident. Other nodes which are behind the first hop of the source node would get the message from the first hop, the third hop from the second hop etc. But if there are nodes which were driving in parallel then they would got the message at the same time. If the accident is already happened and needs a help from the closest transportation and accident management office then the nodes which are located close to the office would transfer the message to it.

To increase the cooperation among nodes and stimulate selfish nodes the amount of credit paid or rewarded per packet had to be higher. Therefore the selfish nodes would not get any benefit other than keeping its previous resources and while looking cooperative nodes with their advantage for their cooperation then in the next time the selfish nodes would became cooperate on the network and got a reward. So in such a fashion we could minimize the rate of selfish nodes. The government or the SP (service provider) had to reward to cooperative nodes.

2. Experimental Procedures, Materials, and Methods

\subsection{General Approach}

As a general approach this study would use quantitative research approach. The reason why we used the Quantitative researcher approach is that we would do our research experimentally by using a collaborative network and traffic simulation tool.

\subsection{Data Source}

As researchers of [49] said, the mobility models occurred in current simulators is not an accurate representation of real vehicle mobility. Then they used real maps, in the TIGER format which is located at (http://www.census.gov/geo/www/tiger) and vehicles move along the streets. Every car moves fully independent of other cars, with a constant speed randomly chosen. The TIGER files contain detailed geographical information about all the roads in a region, from large highways to small streets. But, the TIGER database might not fulfill some traffic-specific information, like traffic control systems (traffic lights) or the number of lanes. Therefore added some extra information is crucial. Other researchers like [50] suggested the data source for the simulation mainly CRAWDAD project which provides a repository where numerous real world traces are available. But as the simulation tool by itself fulfills the above basic elements (number of lanes, lane changing capability, RSU, OBU, traffic lights etc.) that a mobility model should integrated, then we are drawn the road map of Gondar town that we got from Gondar town municipality by using the NCTUns GUI road construction option with the real road map of the town.

\subsection{Message Forwarding Methodology}

The pheromone message is visible for all Ants and they can take an immediate measurement by using the broadcasted message. Even if there is a warning pheromone and food source locating pheromone whatever the pheromone is we are interested on their way of message broadcasting for their common interest. Even they can establish pheromonal trail with the presence of obstacles with no selfish Ants [6]. So every community of Ant can be well informed because of the pheromone broadcasted on their path [2]. Therefore there have to be the mechanism that can decrease the selfishness of human beings and increase our cooperative. That is rewarding drivers that used the way of message broadcasting of Ants for their common goal especially in accident related messages so as to attract the selfish drivers.

\subsubsection{Message Broadcasting Algorithm}

Algorithm 1: Emergency message broadcasting

Data: When accident occurred on the street that the vehicle moves then a cooperative node broadcasts the message for every nodes that are located within 250 meter radius and to RSUs so as to avoid multi car collision (accidents that occurred due to the committed accident because of lack of emergency message) and getting a quick help from responsible bodies, so in the location 1 , the node 1 checks the freshness of the accident and if it is fresh then broadcasts the message.

\section{Begin}

2 if a DTN node 1 is a cooperative node in broadcasting accident messages

Then

31 checks the freshness of the accident

4 If the accident is fresh.

Then

$5 \quad$ N1 broadcasts the message

6 Else

$7 \quad$ N1 drive without broadcasting/because they already got a help and the road is free 
End

$8 \quad$ else

$9 \quad 1$ is a selfish node in broadcasting accident messages

Then

10 N1 drive without broadcasting the accident message

11 end

12 end

Adopted from [56] and edited.

\subsection{Selfishness Detection Method}

Our selfishness detection method was watchdog system, in which the RSU controls whether nodes are cooperative or not because the RSU knows where the nodes were located and in which path they were drive and what messages they broadcasted.

\subsection{Rewarding Model/Incentive Scheme}

The rewarding or incentive schemes so far includes reputation-based schemes, in which the forwarding task is assigned to nodes depending on their reputation level that is, when a node provides services for other nodes, it obtains a good-reputation value like receiving services from other nodes, but if uncooperative nodes eventually detected and excluded from the networks, similarly, in TFT (tit-for-tat) -based schemes, every node forwards messages to a neighbor, based on how many messages the neighbor forwards to it [1]. According to some researchers like [10] proposed to use both credit-based and reputation-based incentive as a single scheme to achieve fairness. In which if and only if the bundles arrive at the destination node, the intermediate participant nodes can get credits from the source node. Even if, the bundle is failed in the middle of the transmission, then the intermediate forwarding nodes still can get good reputation values. Unfortunately the previously described incentive/rewarding schemes, were basically proposed for mobile ad hoc networks (MANET) and may not be suitable for DTNs (delay tolerant networks), for the following reason, in which a common assumption applied in previously described incentive schemes is that, a full end-to-end path between the source and the destination can be determined before data forwarding occurs [20].

However the rewarding/incentive scheme in which we get suitable to our research is paying per packet. But in our case the responsible node for rewarding the cooperative nodes is not the source node, instead the SP or the traffic control bureau collaborating with the government will be responsible therefore finding an end-to-end nodes is not a must. The payment principle is for each successfully transmitted unit-sized packet, each of $\mathrm{N}$ intermediate nodes should receive $\lambda$ credits, whereas the source (the SP in our case) needs to pay $\lambda$ $* \mathrm{~N}$ in total. in addition we need not to determine all the intermediate nodes and we said that accident related bundle are successful if and only if it reaches to nodes around the accident. Means there may not be a single source and destination node instead many nodes can be participated in sending as well as taking the bundle as a destination [9].

\subsubsection{Rewarding Algorithm: Paying Per Packet}

Data: When a cooperative node N1, broadcasts a fresh emergency packet $\mathrm{P}$, then node would rewarded $\lambda$ ( 10 times of its expense) credit for its cooperation. N1 would rewarded.

Total number of $\mathrm{P} *$ total amount of expense for $\mathrm{P}^{*}$ 1 begin

2 if a DTN node 1 is a cooperative node broadcast $\mathrm{P}$ emergency packets

Then

3 the RSU or service provider calculates number of $\mathrm{P}$ and total payment for broadcasting

4 if the total number of $\mathrm{P}$ is 3 and expensed 2 birr for each

Then

5 The RSU or service provider would reward $3 * 2 * 10$.

$6 \quad$ Else

7 no packet is broadcasted and no credits taken from N1

Then

Node N1 would not rewarded

End

8 else

$9 \quad 1$ is a selfish node in broadcasting accident messages

Then

$10 \quad$ N1 got only awareness and cooperative nodes acknowledgement messages

11 end

12 end

Adopted from [10] and edited.

\subsection{Sampling Techniques and Size}

Our sampling technique were a simple random sampling technique in which we selected cooperative, selfish and crushed/broken nodes by using a simple lottery method. Our sample size for all the three simulation was 6,15 and 20 because stimulating selfish nodes with small number of nodes is difficult than large number of nodes ant there is also a more chance of selfish and cooperative nodes reaching on the accident place, if we have more vehicles on the road, then the cooperative node broadcasts the problem and the effect of selfish nodes would be low if we use more number of nodes in the simulation. 


\subsection{Bio-Inspired Literatures}

Swarm intelligence indicates understanding of the collective behavior of animals and insects such as flocks of fishes, ant colonies, swarms of birds or bees and taking this exciting behavior for development of systems. Such systems are made up of simple agents of population interacting directly and indirectly with their environment and with one another.

The current bio-inspired communication and networking protocols as well as algorithms are developed by looking at nature as a source of inspiration, and by adopting the rules and dynamics governing these systems. The natural systems yet help researchers and scholars by providing indicators for mechanisms and solution ways which address the problem that is seen between the limited supply of resources and high demand. Let us take the foraging processes of Ants that, they use their individual inadequate resources for their colonies in order to find food source in an effective way. Due to their cooperative nature of Ants, their foraging process inspired many resource-efficient networking techniques.

Spreading mechanism of Epidemic could be inherited towards efficient information dissemination in highly distributed networks and for opportunistic routing in DTN. Ant colonies, and in general insect colonies, used to perform global activities with no control of any centralized element, could also inspire the development of communication models for wireless networking.

As Bonabeau et al. described "Ant Colony Optimization (ACO) is perhaps the best analyzed branch of swarm intelligence based algorithms". In most cases, swarm intelligence based algorithms are inspired by the behavior of foraging ants. Ants are able to solve complex activities by simple local ways. There is an indirect communication among individuals through modifications of the environment, e.g. pheromone trails are used for efficient foraging. As it is also stated by researchers like As Bonabeau et al. Ants are considered as the "grand masters" in exploration and search. Even in Ant-Bee routing algorithm the researchers used Ants natural behavior for searching of forges and Bees for collecting the forages this indicates not only Ants are better in searching than bees and other social insects but also ants are better in broadcasting or telling the search result to their society. As the name ACO (Ant colony optimization) indicates, the algorithm works on the principles of the foraging process of ants. Naturally Ants perform a random search or random walk for food around their nests and they back to their nests, they marking their route with a pheromone trail. If successful, the ants are returning to the nest by following their own pheromonal trail. While returning, Ants drops an extensive pheromone trail pointing towards the food source. The ants therefore communicated based on their pheromone trail.

As journals stated Perhaps the best known examples of ACO in networking are the AntNet which is done by Di Caro et al. and AntHocNet which is done by Di Caro et al. routing protocols. Both protocols follow the principles of ant routing. In particular, agents are used to simultaneously explore the network and exchange collected information in the same way as ants explore their environment. The key objective of these explorer agents of AntNet is to find a minimum cost path or a shortest path, between the source and the destination, and to update the path-related routing entries in the network [4].

When we see algorithms and schemes that are developed based on the behavior of honey bee includes BEEINFO, which is an Interest-based Forwarding Using Artificial Bee Colony for Socially-aware networking, by Feng Xia et al. and BeeAdHoc, a new routing algorithm in mobile ad hoc networks which proposed to routing using energy efficiently. As we described the algorithms above are inspired by the foraging principles of honey bees. BeeAdHoc is a reactive source routing algorithm and it needs a minimum amount of energy as compared to existing works because it utilizes less control packets to do routing. The algorithm is straightforward and primarily needs two types of messages for routing: scouts, which discovers new routes to the destinations and forgers, which transport data packets and simultaneously evaluate the quality of the discovered paths [12].

In a distributed systems precise synchronization in is a complicated issue and very difficult to achieve. Then new clock synchronization models have been proposed inspired by Fireflies synchronization principles. Furthermore, synchronization principles of fireflies could be applied to the design of communication protocols and also time synchronization protocols which requires precise time synchronization. Activator-inhibitor systems may be exploited for distributed control of sensing periods and duty cycle of target tracking sensor network [4]. As stated by the same author the term Artificial Immune System (AIS) refers to a term that indicates to adaptive systems which is inspired by experimental as well as theoretical immunology for problem solving . AIS, is inspired by the principles and processes of the mammalian immune system, which is to capably detect changes in the environment from the normal system behavior in complicated problem domains. The 
mammalian immune system can protect the body from infections by continuously scanning for invading pathogens. AIS based algorithms typically use the immune system's characteristics of self-learning and memorization. In nature, two immune responses were identified. The first one is to launch a response to invading pathogens leading to an unspecific response. The second immune response remembers previous encounters means it represents the immunologic memory. Even an Artificial Immune System model which used for detection of misbehavior in MANETs is described by [37].

\subsection{Simulation Tool}

The NCTUns (National Chiao Tung University Network simulator) network simulator and emulator is, an open source a high-fidelity and extensible network simulator capable of simulating various devices and protocols used in both wired and wireless networks. Its basic technology is based on the kernel-reentering simulation methodology invented by Prof. S.Y. Wang at Harvard University in 1999. Because of this new methodology, NCTUns provides many basic advantages that can't be easily achieved by previous network simulator such as OPNET Modeler and ns-2 According to the download user database of NCTUns only up to 2010 G.C., 16,246 people from 137 countries downloaded NCTUns software and as of [52] these numbers are still growing. At the beginning, NCTUns was developed for the FreeBSD operating system. But as the Linux operating system is becoming popular, NCTUns now only supports the Linux operating system. Particularly, the Linux version that NCTUns 6.0 (latest version of NCTUns software) currently supports is Red Hat's Fedora 12.

NCTUns 1.0 was originally developed as a network simulator with basic network simulation capabilities. But after the release of NCTUns 4.0, NCTUns adds traffic simulation for example like road network construction and a common vehicular mobility models with its existing network simulation, strongly integrates them together, and provides a fast feedback loop between them. The unique capabilities of NCTUns enables users to study advanced ITS applications in which a vehicle needs to change its moving behavior immediately after receiving a message from a neighboring vehicle or from the infrastructure network to accomplish this capability according to [49] An integrated simulator is needed and according to [47] to have the lane-changing capability, a platform must support multi-lane roads and more intelligent vehicular driving behaviors in the used vehicle mobility models. Example, without this capability, if a vehicle breaks down on a multi-lane road, the vehicles behind it can only stop because no lane-changing operation can be used. However, in the real life, vehicles can change lanes to avoid this broken vehicle. Then due to these added capabilities NCTUns is a useful simulation platform for wireless vehicular ad hoc network researches. The tool supports up to 4096 nodes inside a single simulation with a strong GUI (graphical user interface) support. in version 6.0 the ITS simulation i.e. DVB RCTS satellite radio, Network road construction, Driver Behavior Model, RSU (Roadside unit) Simulation, Onboard unit (OBU) device equipped with, IEEE 802.11(b) both Ad hoc mode and infrastructure mode, GPRS radio etc.

According to the result of the survey at [49] the researcher makes sure that NCTUns is a best simulator than all other simulators, because of its support of different features, easy to deploy to the real world environment and its realistic traces. Due to its Seamless Integration of Emulation and Simulation NCTUns can be turned into an emulator easily. In emulation, nodes in a simulating network can exchange real packets with real-world machines through the simulating network. That is, the simulating network is unknowingly integrated with the real-life network so that simulated nodes and real-life nodes can exchange their packets across the integrated simulated and real-life networks. This capability is very useful for testing the functions and performances of a real-life [53].

Table 1: Experimental Setup / Simulation Setting

\begin{tabular}{ll}
\hline Parameter & Value \\
Number of nodes & 6,15 and 20, (Randomly chosen) \\
\hline Total simulation & 30 times of each simulation \\
$\begin{array}{l}\text { Speed of nodes } \\
\text { Transition coverage/ }\end{array}$ & $250 \mathrm{~km} / \mathrm{h} \sim 50 \mathrm{~km} / \mathrm{h}$ \\
Simulation area & \\
& Freeway mobility model in which we \\
Mobility model & $\begin{array}{l}\text { constructed by using } \\
\text { road construction capability of }\end{array}$ \\
Message size & NCTUns. \\
Routing protocol & Broadcasting \\
Simulation Platform & NCTUns 6.0 \\
Operating system & Fedora 12 \\
\hline
\end{tabular}

Deploy the OBUs and RSUs on the road [48]. Each vehicle recognizes each other by means of $802.11 \mathrm{p}$ standard and GPS. As [47] and other researchers used 250 meters as the upper bound of distance in urban scenarios for $802.11 \mathrm{p}$ communication, so we have chosen the same limits. The number of nodes, speed of nodes and the message sizes described in the setup value are also used by incentive scheme researchers like [9], [10] and [33]. The reason why we used minimum number of nodes, $(6,15$ and 20) in our simulation was that to analyze the effects of selfish nodes we had to start from a small number because the 
occurrences of selfish nodes in a small number of total nodes has high probability of reaching to the accident place alone and if there are no cooperative nodes around, then its negative impact would be high as of [20] and as we checked too. We simulated each individual simulation 30 times, which means simulation 1 was tested 30 times by changing parameters and settings and the same is true for simulation 2 and 3 because researchers at [56] used the same limitation and had a good result. The road map of Gondar town is taken from Gondar town municipality. Because of all vehicles are the same in the simulator, then we randomly chosen the nodes for our simulation sample.

\subsection{Experimental Procedure}

\subsubsection{A general Simulation Setting}

Since there have to be a communication between dispatcher and coordinator then we have to insert the correct loopback address. On the job dispatcher IP address write 127.0.0.1 and 9800 one port address space. At the user information we insert the NCTUns user name and password that we created before.

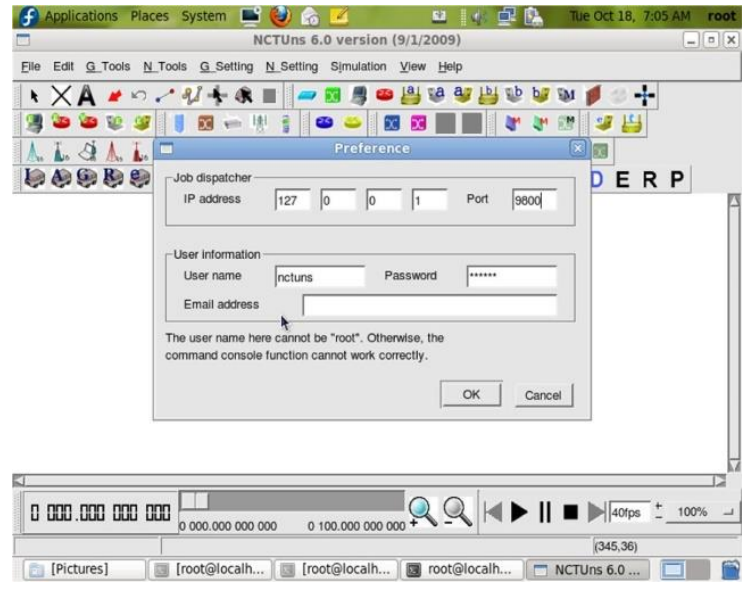

Figure 1. Setting the IP address of job dispatcher

To manage vehicles movement and message broadcasting we would follow the following:

We fill in the options Max_X, Max_Y and Max_Z in meter. It indicates the distance that the node can cover in three directions in which we prefer 3000 meter.

We configure cars profile, (profile1, profile2, profile3, profile4, profile5), $20 \%$ to all the spaces. $20 \%$ to all profiles means each of the profiles would be used by $20 \%$ of the total vehicles. Each profile contains MAX_speed, MAX_acceleration and MAX_decceleration of vehicles.

As we described we use RSUs as a SP (service provider) and we can deploy it manually on the draw topology mode either after finishing these all the above steps or during deploying ITS cars stage. To have a communication between the $802.11(\mathrm{P}) \mathrm{OBU}$ and the RSUs, the RSUs have to be also 802.11 (P) RSU because the automatically deployed vehicles are an agent controlled 802.11 (P) OBU vehicles. To have a communication between OBUs and RSUs, after deploying both OBUs and RSUs on the "draw topology" or " $D$ " mode:-

We used the following car agents for our simulation.

a) CarAgennt_Broken, which used to create a car accident.

b) CarAgent_LaneSwitch, to change a lane during mobility.

c) CarAgent_Down, to make the car stop near to the broken car etc.

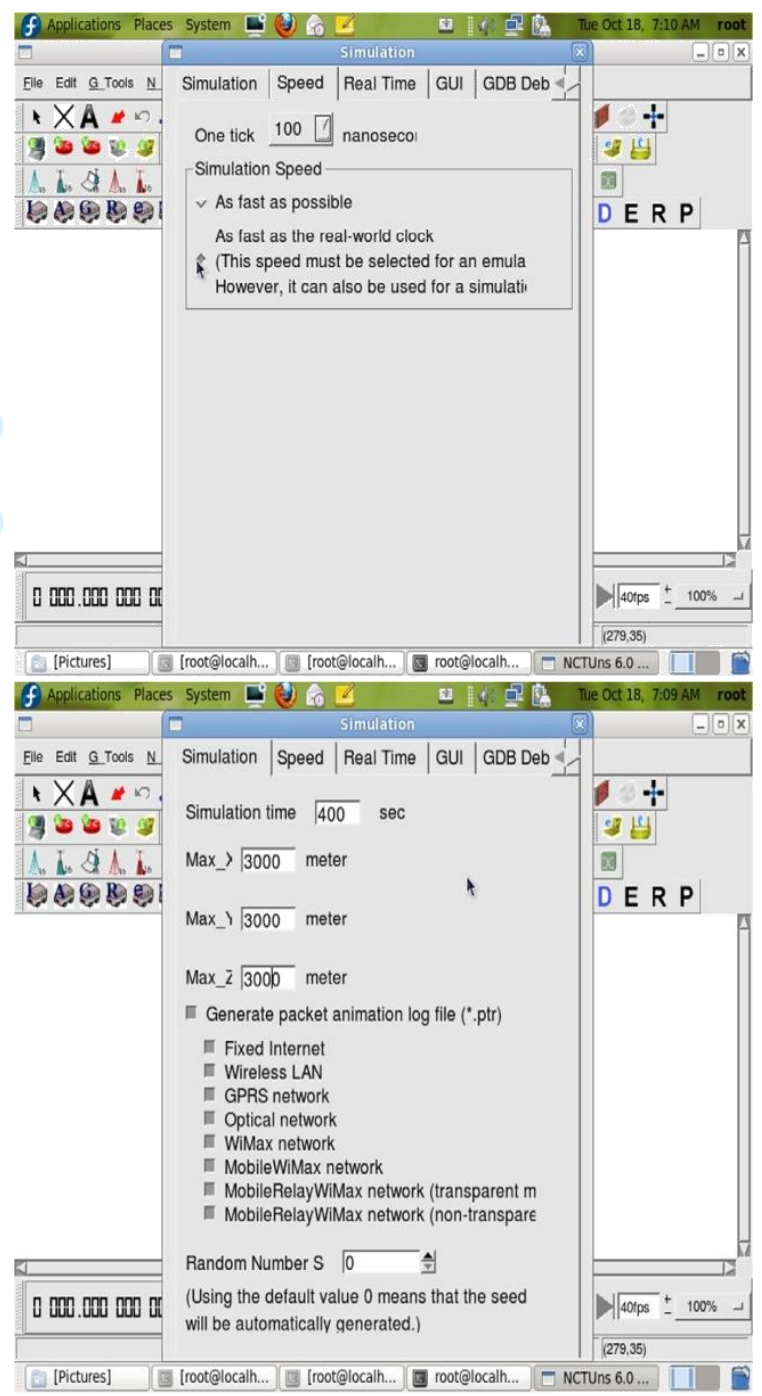

Figure 2. Simulation Area, speed, real time setup

To manage the physical layer properties, at the Transmission power the default is $28.8(\mathrm{dbm})$ we change it to $15 \mathrm{dbm}$ because the simulator was used this amount for 500 meter but we only want 250 meter data transmission area, and the receiver sensitivity is - 
$41.0(\mathrm{dbm})$ again we took half of the default to receive data in a 250 meter distance. But we used the default for the RSU because it has to cover 500 meter.

\section{Results and Discussion}

\subsection{Simulation Results}

\section{Simulation 1}

On the road for the first simulation there would be a randomly selected $6 \quad 802.11(\mathrm{p})$ vehicles and 1 802.11(p) RSU simulated for about 350 seconds.

- The RSU broadcasts 6 awareness and stimulation messages throughout the simulation and rewarding messages to cooperative nodes.

- The reward is 10 times of the cost that the vehicles consumed during broadcasting.

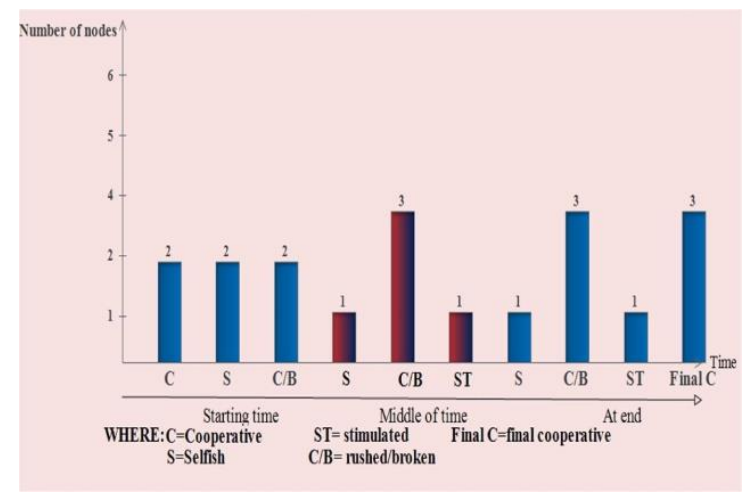

Figure 3. Simulation 1 result

At the start time the first three shapes in the chart indicated that the total number of cooperative, selfish and crushed/broken nodes, at the first stage of the simulation, the next three indicates the remaining total number of selfish, crushed/broken, and stimulated or a node that became a cooperative node. Then at the final stage it indicated that one selfish node was remain as selfish means no additional stimulated node was occur after the middle of the simulation. Therefore the total cooperative node would be 3 because we were having the total of 2 cooperative nodes starting from the beginning of the simulation. But due to one of the cooperative node was crushed/broken at the middle of the simulation it is also added to the crushed/broken nodes. Means one of the cooperative node is counted as both cooperative and crushed/broken node.

The percent indicates the total percent of a given nodes at a given time but because of some of the cooperative nodes may be broken/crushed then they are counted both as a cooperative nodes and crushed/broken nodes since even if they are broken but they were a cooperative nodes too. That is why at the above table we had 50\% cooperative, $16.7 \%$ selfish and 50\% crushed/broken nodes. If we add both it will be more than $100 \%$ but it was due to one cooperative node were broken at the middle of the simulation and counted both as a crushed/broken and as a cooperative node. As the table indicated too, the percentage of accident was increased as of cooperative nodes, so this indicates we can only minimize and avoid accidents related to lack of emergency message but accidents due to other means could be occurred.

Even if one of the cooperative node broken around the end of the simulation it was not due to the lack of emergency message and one of the selfish node became a cooperative. There were 6 OBU nodes or vehicles in the first simulation. Node 10 and 11 were randomly selected to be a selfish or uncooperative node. The random selection method is simple lottery method in which we wrote the ID of all of the nodes and picked the lottery then, the first two picked lottery were considered to be a selfish nodes. So in the case of simulation one (I) node 10 and 11 were selected as a selfish nodes at the starting of simulation and during simulation time node 11 were stimulated and became a cooperative node. Due to its cooperation then it is rewarded and acknowledged by the RSU in which it is considered as a service provider or we can say the country transportation office collaborated with telecommunication. But node 10 remained selfish due to node 10's selfishness factor is more than the reward given by the service provider and not stimulated by the awareness and acknowledgement messages broadcasted by the service provider. As researchers in the area agreed that we can't stimulate all nodes using the same incentive amount and $100 \%$ vehicles can't be cooperative as of [10] we can't avoid selfishness totally and a small fraction of selfish nodes may be remain if we use the same incentive method.

Always while nodes broadcast a message they would add their ID number and this made easy to know and detect which nodes are cooperative and which are selfish. The messages sent by the cooperative nodes were the same for the same accident except they change their own ID.

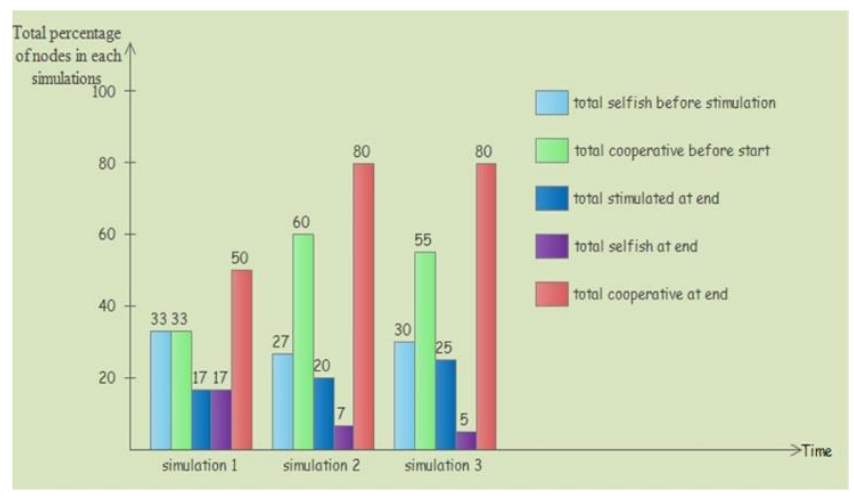

Figure 4. The three simulation comparison chart 
The above figure shows that the total remained selfish nodes decreased as the total participant nodes and the total reward per packet increased in other words their effect decreases as the number of total participant and total reward per packet increased. Therefore the effect of one selfish node in the total of 6 nodes is higher when it compared to one selfish node in 15 and 20 total nodes. Finally stimulating selfish nodes in total small number of nodes and less reward per packet is difficult than that of large nodes. There is also a more chance of selfish and cooperative nodes reaching on the accident place if we have more vehicles on the road then the cooperative node broadcasts the problem and the effect of selfish nodes would be low.

\subsection{Discussion}

The general goal of the paper was to employ a bioinspired reward-based message forwarding for VSN so as to minimize and avoid car accidents that were occurred due to selfish drivers. Both of the simulation results showed that we could stimulate the selfish nodes by rewarding and creating awareness.

Rewarding method by itself had a very high impact on our result because most of the previously done researches related to incentive methods in vehicular social network, were not a paying per packet type instead the source node would pay the intermediate nodes. And some of the researchers in this area suggested that the paying per packet type on rewarding method is not recommended for a DTN (delay tolerant networks) due to the network is intermittent while nodes are moving. But in our research the RSUs or the service provider are responsible bodies for rewarding cooperative driver. This RSUs can both received and transmitted up to 500 meter radius but nodes could only receive and transmitted in 250 meter and as soon as the cooperative nodes broadcasted an emergency message then they would be rewarded and acknowledged before they leave the limited distance.

Researchers at [10] used Credit-based incentive as well as reputation based incentive in which only a participant that had high credit could be rewarded and the other would got good reputation but the participants or an intermediate node have to be rewarded if they was participate in the broadcasting if not may be not participate for future broadcasting. Researchers at [33] used weighted rewarding almost the same with the above incentive method but this scheme didn't consider giving good reputation for intermediate nodes again it demotivated intermediate nodes that participated in broadcasting but had no high weight or winner. Researchers at [9] used profitsharing model in which the intermediate nodes profitsharing model, means that the intermediate nodes involved in a successful bundle delivery would paid with a dividend of the total credit provided by the source node. A very good incentive scheme but we believe if source node should pay for all cooperating nodes then the source node may become a selfish node for saving of his/her resources and even the incentive given by a source node may be minimum or less that the selfishness factor of selfish nodes. But the above researchers believed that rewarding cooperative nodes can stimulate selfish nodes and they showed the effectiveness of their result by using simulation. In the reverse of the above researchers there are also researchers who believes that punishing selfish nodes especially excluding it from the network could be the solution but they forgot that rewarding changes people to become good behave than punishment and excluding them has no any advantage other than punishing them. In this side researchers at [39] are examples. Therefore our research was agreed with ideas of researchers at [9], [10] and [33], in which they were believed rewarding is better and they simulated and proved their result but we changed the rewarding method because we got paying per packet is the best incentive method as we tried on our simulation.

Finally our result showed that we can stimulate the most numbers of selfish nodes by rewarding cooperative nodes by using paying per packet method. In addition to rewarding nodes awareness creation and broadcasting the acknowledgement messages to all of the nodes in the network. In all of the three simulations we stimulated selfish nodes and also there were selfish nodes that their selfishness factor were more than that of our result and we suggest that increasing the reward as much as possible is advisable to stimulate additional number of selfish nodes.

\subsection{Interpretation}

Our result showed that at the first simulation we were had $33.3 \%$ of cooperative and $33.3 \%$ selfish nodes means equal number of cooperative and selfish nodes but at the end of the simulation the selfish nodes decreased by half means $50 \%$ of the selfish nodes became cooperative and at the same time the number of cooperative nodes increased to $50 \%$ of the total nodes and it means from $33.3 \%$ to $50 \%$ and the increment was $16.7 \%$.

On the second simulation we decreased the proportion of selfish nodes to that of total cooperative nodes at the beginning of the simulation that was from the total percentage of nodes $60 \%$ were cooperatives and $26.7 \%$ were selfish nodes and finally the cooperative nodes increase to $80 \%$ means increment by $20 \%$ and the selfish nodes decrease by $20 \%$ to the reverse of cooperative nodes. From the total number of 4 nodes 
or $26.7 \%$ of nodes three of them became cooperatives means $75 \%$ from the total selfish nodes became cooperatives due to the rewarding given by the RSUs or the service providers and the acknowledgement and awareness messages broadcasted by the service providers. On the third simulation there were $55 \%$ cooperatives and $30 \%$ of the nodes were selfish nodes from the total number of nodes. Finally the total cooperative nodes became $80 \%$ means increased by $25 \%$ and the selfish nodes became 5\% means decreased by $25 \%$. The selfish nodes from the total number of selfish nodes $83.3 \%$ of them became cooperatives and only $16.7 \%$ of the selfish nodes remained selfish.

Throughout the three simulations the number of accident increased as the number of total nodes increased in the same simulation distance and area. As the number of nodes participated on the area increases the probability of having an accident increases and the probability of stimulating selfish nodes increased using the same incentive method and increased amount because of there is a probability of accident commit increases and the rewarding and acknowledgement messages increases in which this could stimulate the selfish nodes. Therefore the number of future accidents that were occurred due to selfish nodes will be decreased and due to emergency messages could be reached to service providers then people at the place of accident will get fast help from the responsible bodies. But we can't totally avoid accidents that could be committed by other means for example technical problem, road problem, lack of experience of drivers etc.

\section{Conclusion and Recommendation}

\subsection{Conclusion}

The Ants capability of working together and their way of message transmission makes us inspired especially their cooperation for their common goal and dropping down pheromones which used as a trail for themselves and for others. Therefore by keeping this Ants behavior of cooperation and pheromonal message forwarding, we would use for drivers cooperation and message forwarding. VSNs became particularly useful in an emergency occasion. Such emergency-based VSNs might serve as a way for people to ask for and provide assistance to each other in critical situations like road accidents and street assistance. There might also be VSNs established to communicate roadway events such as accidents, congestions etc., As researchers indicated, Broadcasting is a necessity for VANETs not only for forwarding but also for delivering information without constructing a data path. Broadcasting is an effective model in safety- related information dissemination to achieve cooperative driving in vehicular ad hoc network (VANET).

The majority of the existing routing protocols have an unreasonable assumption that was, they assume "all nodes in the network are unselfish". Means, every node in the network is willing to receive and transmit the messages sent by other nodes. But in the real world, majority of the people are rational and may be selfish. Our selfishness detection method was watchdog, in which the RSU controls whether nodes are cooperative or not because the RSU knows where the nodes were located and in which path they were drive and what messages they broadcasted. To increase the cooperation among nodes and stimulate selfish nodes the amount of credit paid or rewarded per packet had to be higher. Paying per packet would use as a rewarding model. We care on the rewarding model because the design of the reward calculation is the core of the incentive scheme. Fairness is guaranteed in which every node which participated in emergency message forwarding was rewarded and nodes were rewarded based on their total delivered packets means nodes which transmitted 3 packets and 10 packets were not rewarded the same amount, instead $3 * \operatorname{cost}$ for a packet*10 and $10 *$ cost for a packet*10.

Whatever we care about incentive schemes the first job should be detecting the selfish node from the whole nodes in the network in order to combat selfishness. An incentive scheme or popular charging method called paying per packet, which means that, for each successfully transmitted unit-sized packet, each of $\mathrm{N}$ intermediate nodes should receive $\lambda$ credits, whereas the source (the SP in our case) needs to pay $\lambda * \mathrm{~N}$ in total, Which was we selected for our research.

A field trial of wireless vehicular communication research usually includes a large number of vehicles and people like drivers and computer operators for generating acceptable results. Conducting such field trials is very costly since, many communication equipment need to be purchase, many vehicles need to be purchase or rent, and many experimenters need to be employee. Even, during high-speed scenario, the experimenters may face potential dangers such like collisions with pedestrians or vehicles. Moreover, it is very complex to accurately control and repeat a real world field trial on the roads, which is terrible for debugging the problems and enhancing the performances of a new protocol.

As a general approach this study would use quantitative research approach. The reason why we used the Quantitative research approach is that we have done our research experimentally by using a collaborative network and traffic simulation tool. Our 
sampling technique were a simple random sampling technique in which we selected cooperative, selfish and crushed/broken nodes by using a simple lottery method. Our sample size for the three simulations was 6,15 and 20 sequentially, because stimulating selfish nodes with small number of nodes with less reward is difficult than large number of nodes and more reward, there is also a more chance of selfish and cooperative nodes reaching on the accident place, if we have more vehicles on the road then the cooperative node broadcasts the problem and the effect of selfish nodes would be low.

Experimental methodologies are widely applied in computer science and related fields to evaluate new solutions for problems. Field trial of wireless vehicular communication research usually includes a large number of vehicles and people like drivers and computer operators for generating acceptable results. Conducting such field trials is very costly and during high-speed scenario, the experimenters may face potential dangers such like collisions with pedestrians or vehicles. Therefore due to the above and other problems, it is highly recommended to use software simulation to perform testing and evaluations prior to conducting field trials.

The data analysis method is a simple descriptive way of analysis in which the result of the simulation should be described in a plain text or English. The unique capabilities of NCTUns enables users to study advanced ITS applications in which a vehicle needs to change its moving behavior immediately after receiving a message from a neighboring vehicle or from the infrastructure network.

Throughout the three simulations the number of accident increased as the number of total nodes increased in the same simulation distance and area. As the number of nodes participated on the area increases the probability of having an accident increases and the probability of stimulating selfish nodes increased using the same incentive method and different incentive amount because of there is a probability of accident commit increases and the rewarding and acknowledgement messages increases in which this could stimulate the selfish nodes. Therefore the number of future accidents that were occurred due to selfish nodes will be decreased and due to emergency messages could be reached to service providers then people at the place of accident will get fast help from the responsible bodies.

\subsection{Recommendation}

Based on the results obtained from the experiment, the researcher makes the following recommendations.
- The country telecommunication and traffic management office have to work with collaboration and start to reward good behave drivers until we start to use the intelligent transportation systems and having $\mathrm{OBU}$ vehicles.

- The traffic management office have to encourage car owners to use the intelligent transportation systems for their vehicles because to reward good behave drivers using our rewarding method the vehicle have to have an OBU devices.

- Since the NCTUns simulator is a popular simulator for vehicle to vehicle communication and its GUI minimizes the wastage of time for program development and embedding federated mobility models then the researcher recommends that other researchers in the area can enjoy the tool with its additional features.

- Security: researchers shall provide solutions for privacy and trust issues.

- Researchers should also experiment by involving or integrating pedestrians with vehicles. Researchers identify the right Incentive model that can include both vehicles and pedestrians.

\section{References}

[1]. Anna Maria Vegni and Valeria Loscri "A Survey on Vehicular Social Networks", IEEE Communications Surveys \& Tutorials, p-1, 2015.

[2]. Johan Billen, "Signal variety and communication in social insects", Zoological Institute, University of Leuven, Belgium, 2006

[3]. Tristram Wyatt, "key roles for chemical communication in animal biology", plenary $7^{\text {th }}$ international conference on comparative physiology and biochemistry, salvador, Brazil, $\mathrm{p}-8,2007$,

[4]. Falko Dressler and Ozgur B. Akan, "A Survey on Bio-inspired Networking", Computer Networks and Communication Systems, n.d.

[5]. Francis L. W. Ratnieks, "Ophistication in the foraging trail networks of Pharaoh's ants", Laboratory of Apiculture \& Social Insects, University of Sussex, n.d.

[6]. Liviu A. Panait and Sean Luke, "Ant Foraging Revisited", George Mason University, Fairfax

[7]. Ben Bowlin, are Ants better communicators than you.mp4 [medium= English], brain stuff, n.d.

[8]. Shu-Chuan Chu, John F. Roddick and JengShyang Pan, "Ant colony system with 
communication strategies", science direct, n.d.

[9]. Haojin Zhu, Xiaodong Lin, Rongxing Lu, Yanfei Fan and Xuemin (Sherman) Shen, "SMART: A Secure Multilayer Credit-Based Incentive Scheme for Delay-Tolerant Networks", IEEE transactions on vehicular technology, vol. 58, no. 8, October 2009

[10]. Rongxing Lu, Xiaodong Lin, Haojin Zhu, Xuemin (Sherman) Shen, and Bruno Preiss, "Pi: A Practical Incentive Protocol for Delay Tolerant Networks", IEEE transactions on wireless communications, vol. 9, no. 4, april 2010

[11]. Alessandro Floris, Michele Nitti, Roberto Girau and Luigi Atzori, "On adding the social dimension to the Internet of Vehicles: Friendship and middleware", ResearchGate, Conference Paper, May 2014

[12]. Horst. F. Wedde, Muddassar Farooq, Thorsten Pannenbaecker, Bjoern Vogel, Christian Mueller, Johannes Meth and Rene Jeruschkat, "BeeAdHoc: An Energy Efficient Routing Algorithm for Mobile Ad Hoc Networks Inspired by Bee Behavior", Washington, DC, USA, June 25-29, 2005

[13]. Nitin Raut and Abdulla Shaik, "Design and Development of Automatic Vehicle accident detection Localization of Automobile Using Bluetooth Technology", International Journal of Advanced Research in Computer and Communication Engineering, Vol. 3, Issue 3, March 2014

[14]. Tibebe Beshah and Shawndra Hill," Mining Road Traffic Accident Data to Improve Safety: Role of Road-related Factors on Accident Severity in Ethiopia", n.d.

[15]. Ministry of Infrastructure Ethiopian Roads Authority, how safe are Ethiopian roads?: from the perspective and timeframe of the Road Sector Development Program , April 2005

[16]. "Ant's surprising life style" The federal ethics and anti-corruption commission vo 14 , No 1 , September 2007 E.C.

[17]. Assamala Amoi-Séminet and Olive Kobusingye Road safety in the whole African region the facts 2013 Made possible through funding from Bloomberg Philanthropies, 2013

[18]. Sourav Kumar Bhoi and Pabitra Mohan Khilar, "Vehicular communication: a survey", Published in IET Networks, 2013

[19]. Stephen Smaldone, Lu Han, Pravin Shankar, and Liviu Iftode "RoadSpeak: Enabling Voice Chat on Roadways using Vehicular Social Networks”, SocialNets'08, (Glasgow,
Scotland, UK), April 1, 2008.

[20]. Haigang Gong, Lingfei Yu, and Xue Zhang, "Social Contribution-Based Routing Protocol for Vehicular Network with Selfish Nodes", Hindawi Publishing Corporation, International Journal of Distributed Sensor Networks, 2014

[21]. C.-F. Chiasserini, E. Fasolo, R. Furiato, R. Gaeta, M. Garetto, M. Gribaudo, M. Sereno and A. Zanella, "Smart Broadcast of Warning Messages in Vehicular Ad Hoc Networks", n.d.

[22]. Subir Biswas, Raymond Tatchikou and Francois Dion, "Vehicle-to-Vehicle Wireless Communication Protocols for Enhancing Highway Traffic Safety", IEEE Communications Magazine • January 2006

[23]. Gokhan Korkmaz, Eylem Ekici, Fusun Ozguner and Umit Ozguner, "Urban MultiHop Broadcast Protocol for Inter-Vehicle Communication Systems", (Philadelphia, Pennsylvania, USA), October 1, 2004

[24]. Jun Luo and Jean-Pierre Hubaux, "A Survey of Inter-Vehicle Communication", School of Computer and Communication Sciences EPFL, CH-1015, (Lausanne, Switzerland), n.d.

[25]. Yuanguo Bi, Hai Zhao and Xuemin (Sherman) Shen, "A Directional Broadcast Protocol for Emergency Message Exchange in InterVehicle Communications", IEEE Communications Society, 2009

[26]. Wai Chen, Ratul K. Guha, Taek Jin Kwon, John Lee and Irene Y. Hsu, "A Survey and Challenges in Routing and Data Dissemination in Vehicular Ad-hoc Networks", Proceedings of the 2008 IEEE International Conference on Vehicular Electronics and Safety, (Columbus, OH, USA) September 22-24, 2008

[27]. A.Malathi and S.Antoinette Aroul Jeyanthi, "a survey on vehicular network", iracst International Journal of Advanced Computing, Engineering and Application (IJACEA), ISSN: 2319-281X, Vol. 3, No. 1, February 2014

[28]. Giovanni Resta, Paolo Santi and Janos Simon, "Analysis of Multi-Hop Emergency Message Propagation in Vehicular Ad Hoc Networks", ACM, MobiHoc'07,( Montreal, Qu ' ebec, Canada), September 9-14, 2007

[29]. Wenshuang Liang, Zhuorong Li, Hongyang Zhang, Yunchuan Sun, and Rongfang Bie, "Vehicular Ad Hoc Networks: Architectures, Research Issues, Challenges and Trends", Springer International Publishing, (Switzerland), 2014

[30]. Greg Bigwood and Tristan Henderson, "IRONMAN: Using social networks to add 
incentives and reputation to opportunistic networks", n.d.

[31]. Jobin Mathew, "Mobility Model for Vehicular Ad Hoc Networks: A Survey and Taxonomy", In partial fulfillment of Requirements in Degree of Master of Technology in computer $\&$ information science, seminar report 20092011

[32]. Yanyan Zhuang, Jianping Pan, Yuanqian Luo, and Lin Cai, "Time and Location-Critical Emergency Message Dissemination for Vehicular Ad-Hoc Networks", IEEE journal on selected areas in communications, VOL. 29, NO. 1, January 2011.

[33]. Feng Li and Jie Wu, "FRAME: An Innovative Incentive Scheme in Vehicular Networks", n.d.

[34]. Lior Strahilevitz, “'How's My Driving?' for Everyone (and Everything?)", University of Chicago Law School, (Chicago Unbound), 2006

[35]. Barrie E. Litzky, Kimberly A. Eddleston, and Deborah L. Kidder, "The Good, the Bad, and the Misguided: How Managers Inadvertently Encourage Deviant Behaviors", 2006

[36]. James Prochaska, John Norcross and Carol Diclemente, "CHANGING FOR GOOD", n.d.

[37]. J.-Y. Le Boudec and S. Sarafijanovic, "An Artificial Immune System Approach to Misbehavior Detection in Mobile Ad-Hoc Networks, in: 1st International Workshop on Biologically Inspired Approaches to Advanced Information Technology", Springer,Vol., (Lausanne, Switzerland), 2004

[38]. Karagiannis G. , Altintas O. , Ekici E. , Heijenk G. , Jarupan B. , Lin K. and Weil, T., "Vehicular Networking: A Survey and Tutorial on Requirements, Architectures, Challenges, Standards and Solutions ", in IEEE Communications Surveys \& Tutorials Vol. 13 , No 4, 2011

[39]. S. Xu, M. Lu, Y. Chen, L. Shu, and X. Gu, "A cooperation scheme based on reputation for opportunistic networks," in Proc. of International Conference on Computing, Management and Telecommunications, 2013.

[40]. J. Douceur and T. Moscibroda, "Lottery trees: motivational deployment of networked systems". In Proc. of ACM SIGCOMM, 2007.

[41]. H. Zhou, J. Wu, H. Zhao, S. Tang, C. Chen, and J. Chen, "Incentive-driven and freshnessaware content dissemination in selfish opportunistic mobile networks", in Proc. of the IEEE 10th International Conference on Mobile Ad-Hoc and Sensor Systems, 2013.
[42]. Y. Zhang, W. Lou, W. Liu and Y. Fang, "A secure incentive protocol for mobile ad hoc networks", Wirel. Netw., vol. 13, no. 5, Oct. 2007.

[43]. Steven M. Ross and Gary R. Morrison, "experimental research methods", n.d.

[44]. Jose Nelson Amaral, "About Computing Science Research Methodology", n.d.

[45]. Yair Levy and Timothy J. Ellis, "A Guide for Novice Researchers on Experimental and Quasi-Experimental Studies in Information Systems Research", Interdisciplinary Journal of Information, Knowledge, and Management Volume 6, 2011

[46]. Yamith J. fandino, Chapter 2, Quantitative, Qualitative, and Mixed Research, internet: httt://www.bcps.org/lis/images/lec2, [accessed $12 / 01 / 2016]$.

[47]. Shie-Yuan Wang and Chih-Liang Chou, "nctuns simulator for wireless vehicular ad hoc network research", n.d.

[48]. Shivani Singh, Dr B.V.R Reddy, "Simulating VANET Scenario in Nctuns-6.0", National Workshop-Cum-Conference on Recent Trends in Mathematics and Computing, Proceedings published in International Journal of Computer Applications, 2011

[49]. Cristian Gorgorin, Victor Gradinescu, Raluca Diaconescu, Valentin Cristea and Liviu Iftode, "an integrated vehicular and network simulator for vehicular ad-hoc networks", n.d.

[50]. S.Y. Wang and C.L. Chou, "NCTUns 5.0 Network Simulator for Advanced Wireless Vehicular Network Researches", Tenth International Conference on Mobile Data Management: Systems, Services and Middleware, 2009

[51]. Ning Lu, Tom H. Luan, Miao Wang, Xuemin (Sherman) Shen and Fan Bai, "Capacity and Delay Analysis for Social-Proximity Urban Vehicular Networks", n.d.

[52]. Shie-Yuan Wang, Chih-Liang Chou, Chih-Che Lin and Chih-Hua Huang, "The Protocol Developer Manual for the NCTUns 6.0 Network Simulator and Emulator", Network and System Laboratory, Department of Computer Science, National Chiao Tung University, [Taiwan], January 15, 2010

[53]. Shie-Yuan Wang, Chih-Liang Chou and ChihChe Lin, "The GUI User Manual for the NCTUns 6.0 network Simulator and Emulator", Network and System Laboratory, Department of Computer Science, National Chiao Tung University, [Taiwan], January 15, 2010 
[54]. Shie-Yuan Wang and Yi-Bing Lin, "NCTUns network simulation and emulation for wireless resource management", wireless communications and mobile computing, 2005

[55]. Jerom Harri, Fethi Filali and Christian Bonnet, "Mobility Models for Vehicular Ad Hoc Networks: A Survey and Taxonomy", IEEE communications surveys \& tutorials, vol. 11, no. 4, fourth quarter 2009

[56]. Feng Xia, Li Liu, Jie Li, Ahmedin Mohammed Ahmed, Laurence Tianruo Yang, and Jianhua $\mathrm{Ma}$, "BEEINFO: Interest-based Forwarding Using Artificial Bee Colony for Sociallyaware Networking", IEEE transactions on vehicular technology, April 30, 2013. 